\title{
Correction to: Effect of Simultaneous Combined Treadmill Training and Magnetic Stimulation on Spasticity and Gait Impairments after Cervical Spinal Cord Injury by Hou, J., et al., (2020). J. Neurotrauma 37, 1999-2013 (DOI: 10.1089/neu.2019.6961)
}

In the September 15, 2020 issue of Journal of Neurotrauma (Vol. 37, No. 18; 1999-2013) the article entitled "Effect of Simultaneous Combined Treadmill Training and Magnetic Stimulation on Spasticity and Gait Impairment after Cervical Spinal Cord Injury" by Hou et al. requires correction. The online version of the article appeared correctly with the images published in color. However, due to a clerical error, the images were published in grayscale in the print version of the journal though they, too, should have been published in color. The images are reproduced below, in color, as they should have appeared. Note that Figure 8 is not reproduced here as it appeared correctly.

The Publisher sincerely apologies to the authors for this error, as well as to the Journal's readership.

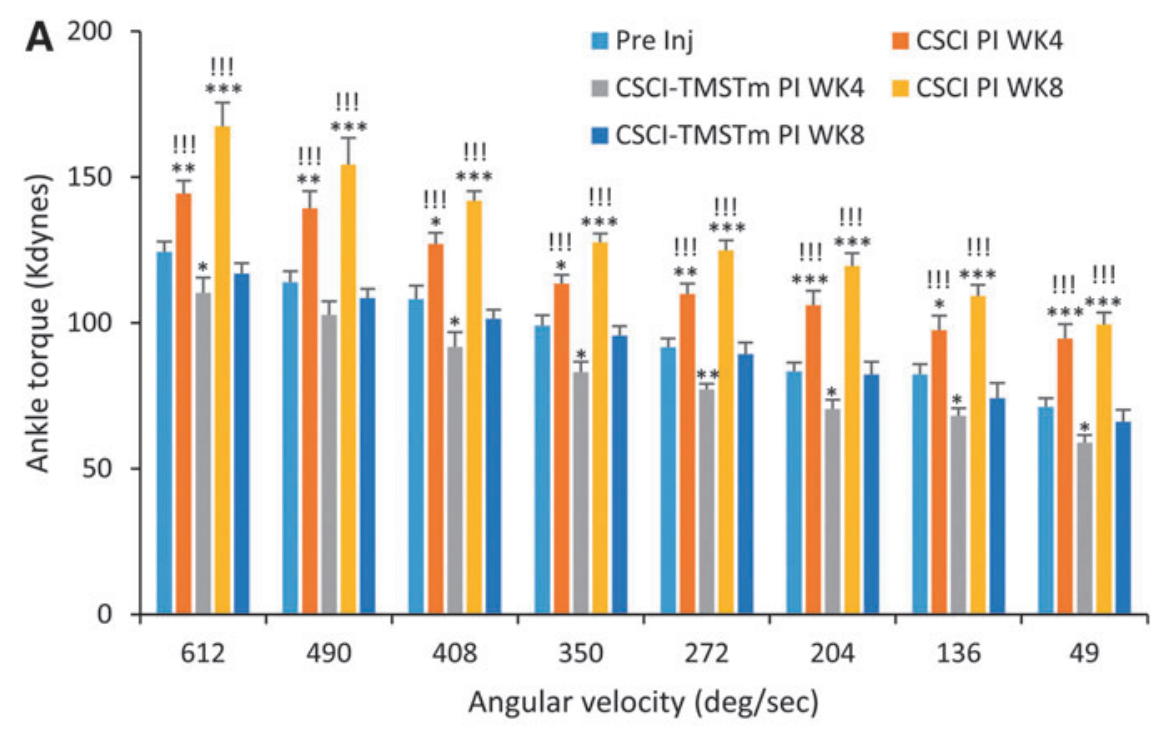

FIG. 1. Velocity-dependent ankle torque (VDAT) (A) and time locked electromyography-root mean square (EMG-RMS) amplitudes (B) of cervical spinal cord injury (CSCI) untreated and transcranial magnetic stimulation/treadmill training (TMSTm) treated animals at pre-injury, post-injury (PI) week 4 and PI week 8 . The CSCI untreated animals showed significantly increased VDAT in all tested velocities at both PI week 4 and PI week 8 when compared with pre-injury and the TMSTm treated group (A). The TMSTm treatment completely blocked the development of CSCI-induced spasticity. The time-locked EMG-RMS also showed a similar trend as VDAT (B). One example waveform of VDAT and time-locked EMG-RMS at the highest test velocity of ankle rotation (612 deg/sec) from each group (randomly selected; normal, CSCI at PI week 8 and CSCI-TMSTm at PI week 8 ) is shown in $(\mathbf{C}) . *, p<0.05, * *, p<0.01, * * *$, $p<0.001$, compared with pre-injury; !, $p<0.05$, !!,$p<0.01$, !!!, $p<0.001$, compared with CSCI-TMSTm. 

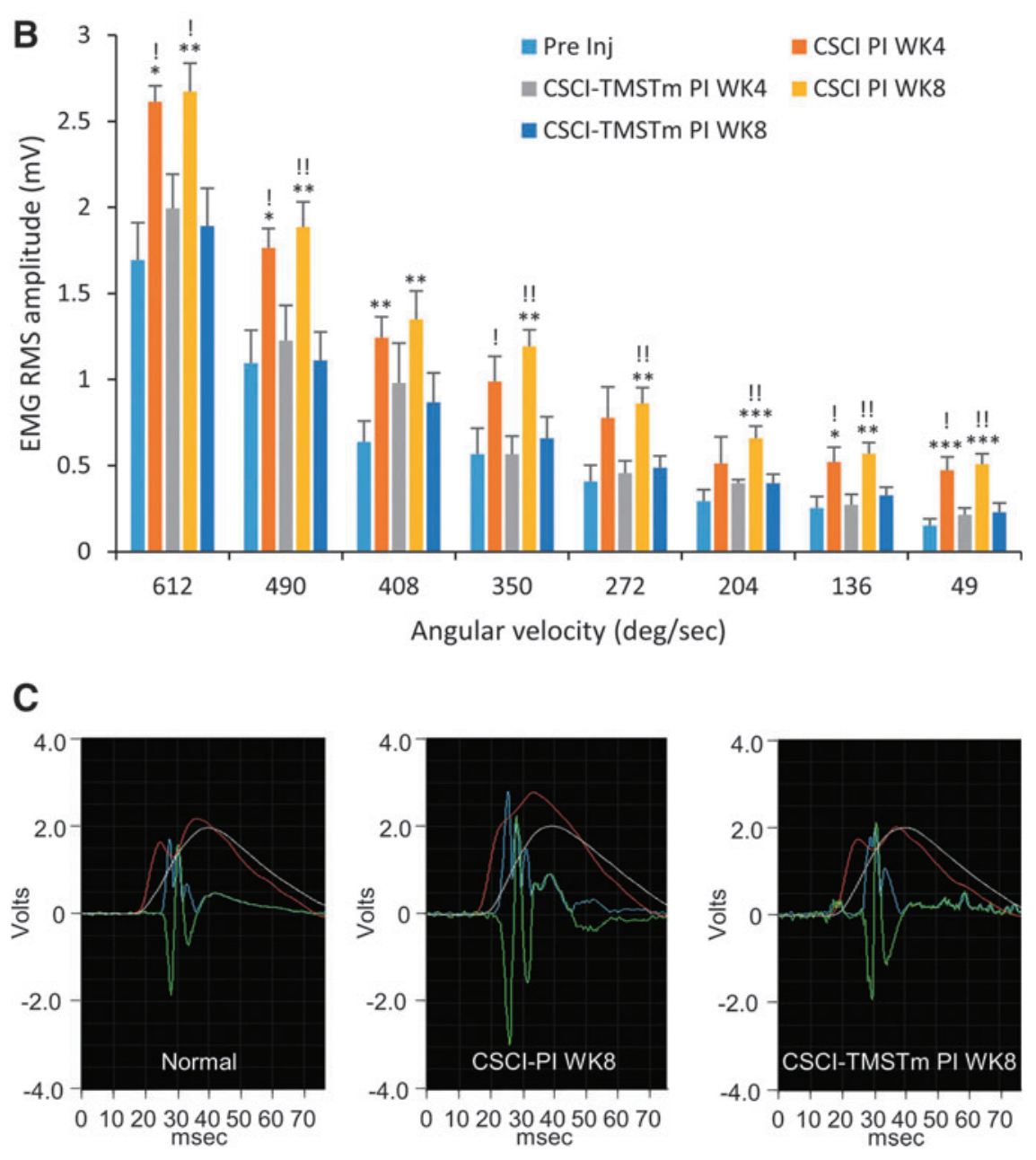

FIG. 1. (Continued)

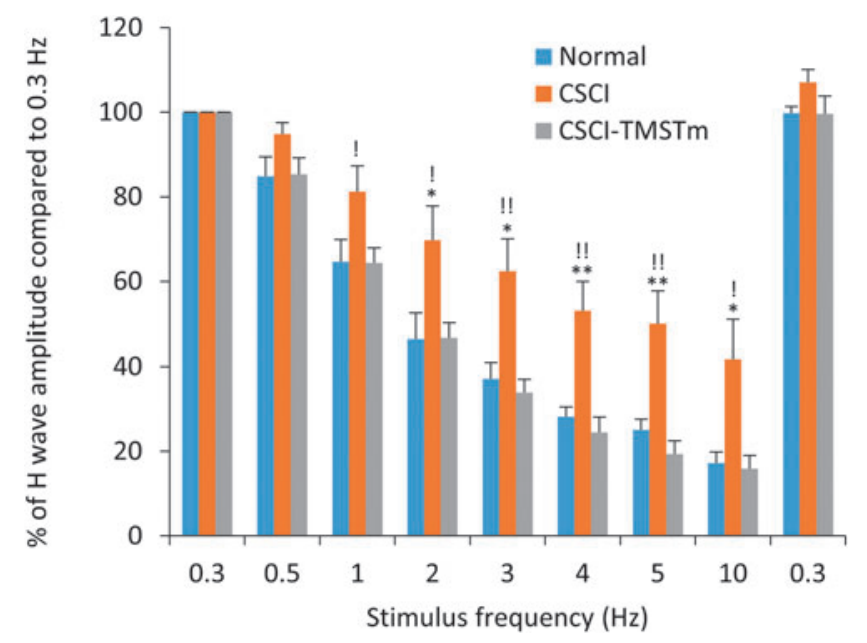

FIG. 2. Rate-depression of the tibial/plantar H-reflexes of normal, cervical spinal cord injury (CSCI), and CSCI-transcranial magnetic stimulation/treadmill training (TMSTm) at post-injury (PI) week 10. AT PI week 10, the CSCI untreated group showed significantly reduced rate-depression compared with normal. The TMSTm combination therapy group revealed complete normalization in rate-depression. *, $p<0.05, * *, p<0.01$, compared with normal; !, $p<0.05, ! !, p<0.01$, compared with the CSCI-TMSTm group at the same testing time point. 


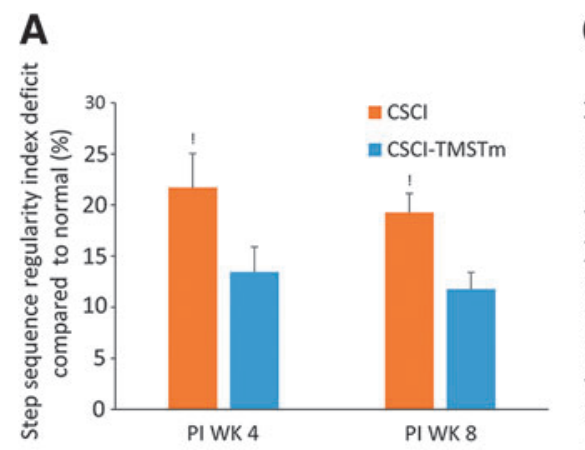

C
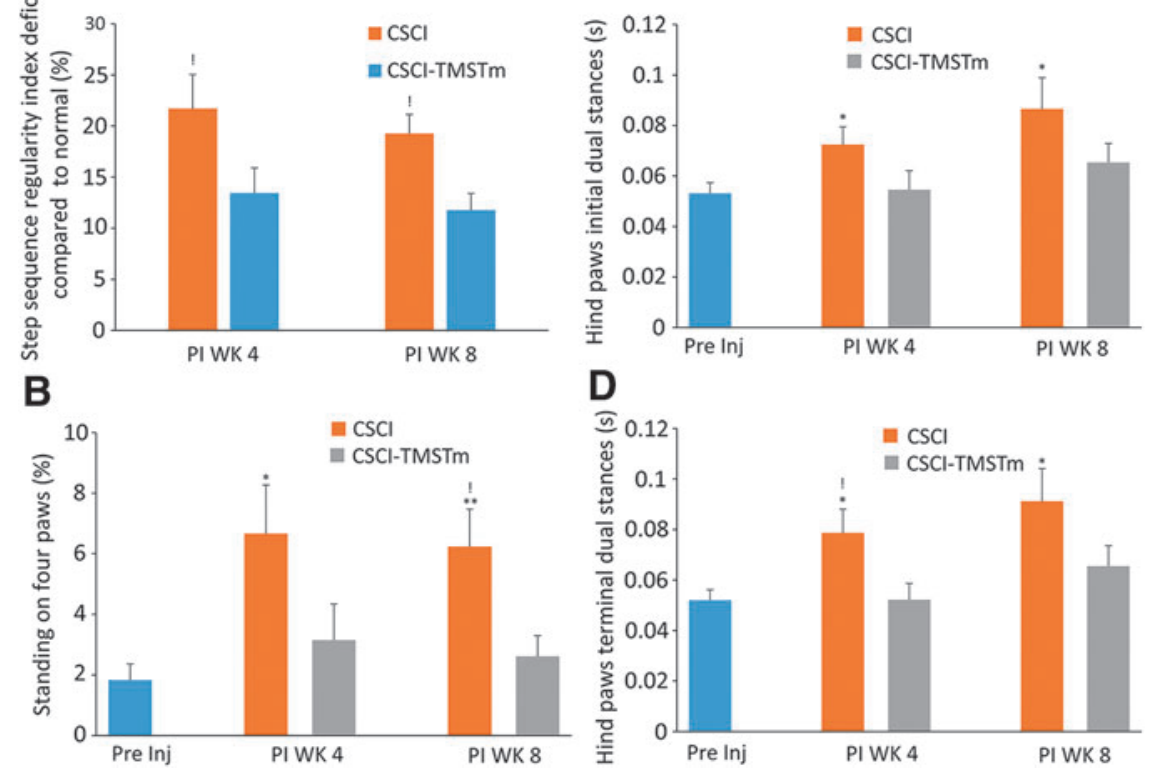

FIG. 3. Catwalk gait analysis was conducted at pre-injury, post-injury (PI) week 4 and PI week 8 . The percentage deficit of step sequence regularity index (SSRI) compared with normal animals at PI week 4 and week 8 is shown in (A). At both time points, the cervical spinal cord injury (CSCI) untreated group showed significantly greater deficit compared with the transcranial magnetic stimulation/treadmill training (TMSTm) group. The percentage in duration of simultaneous standing on four paws during the Catwalk whole testing period is shown in (B). The CSCI untreated group showed significantly greater percentage time of simultaneous four paws contact with the glass plate during locomotion compared with the pre-injury and PI week 8. Normal locomotor parameters were observed in the TMSTm therapy group. The CSCI untreated group also showed significant increases in hindpaw initial dual stances (C) and hindpaw terminal dual stances (D) at both PI week 4 and PI week 8 when compared with pre-injury. The TMSTm group did not show significant differences at any of the tested time points when compared with pre-injury, however. In fact, at PI week 4, the TMSTm trerapy group showed significant decreases of the hindpaw terminal dual stances when compared with the CSCI untreated group. *, $p<0.05, * *, p<0.01$, compared with pre-injury; !, $p<0.05$, compared with CSCI-TMSTm.

A

$\mathrm{CSCl}$

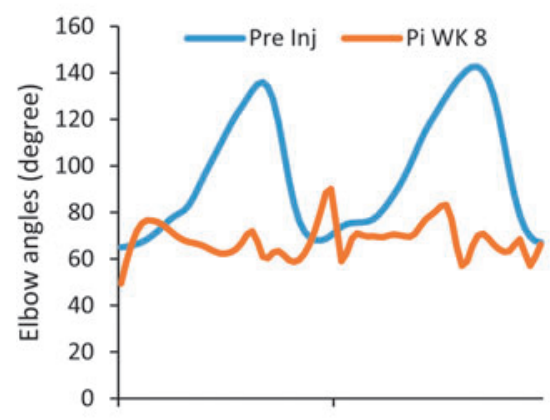

C
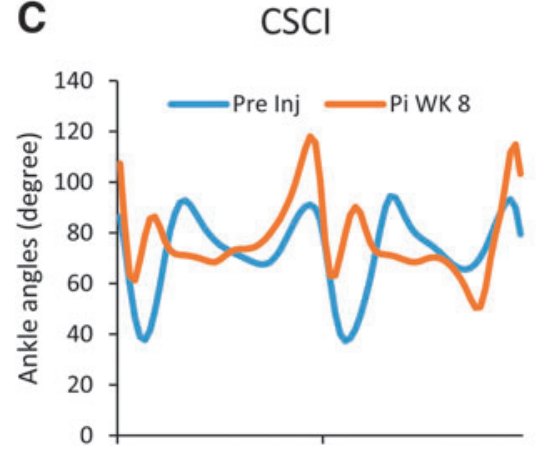

B

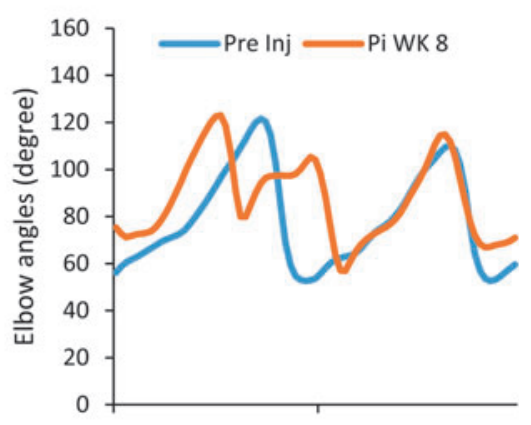

D

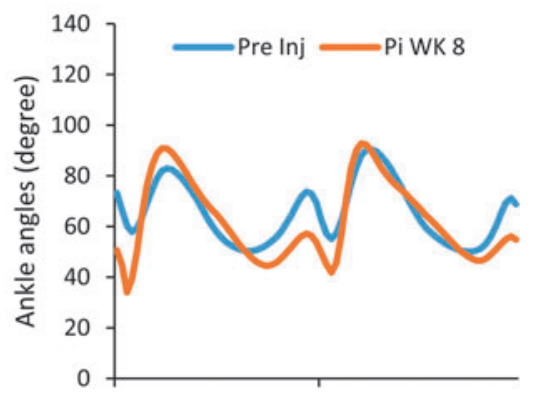

FIG. 4. Elbow and ankle angular displacements are shown during two continuous normalized forelimb (A, B) and hindlimb $(\mathbf{C}, \mathbf{D})$ step cycles of representative cervical spinal cord injury (CSCI) $(\mathrm{A}, \mathbf{C})$ and transcranial magnetic stimulation/treadmill training (TMSTm) (B, D) rats at pre-injury and PI week 8 . The TMSTm treated animals showed more normal patterns in both angular displacements than the CSCI untreated group. 

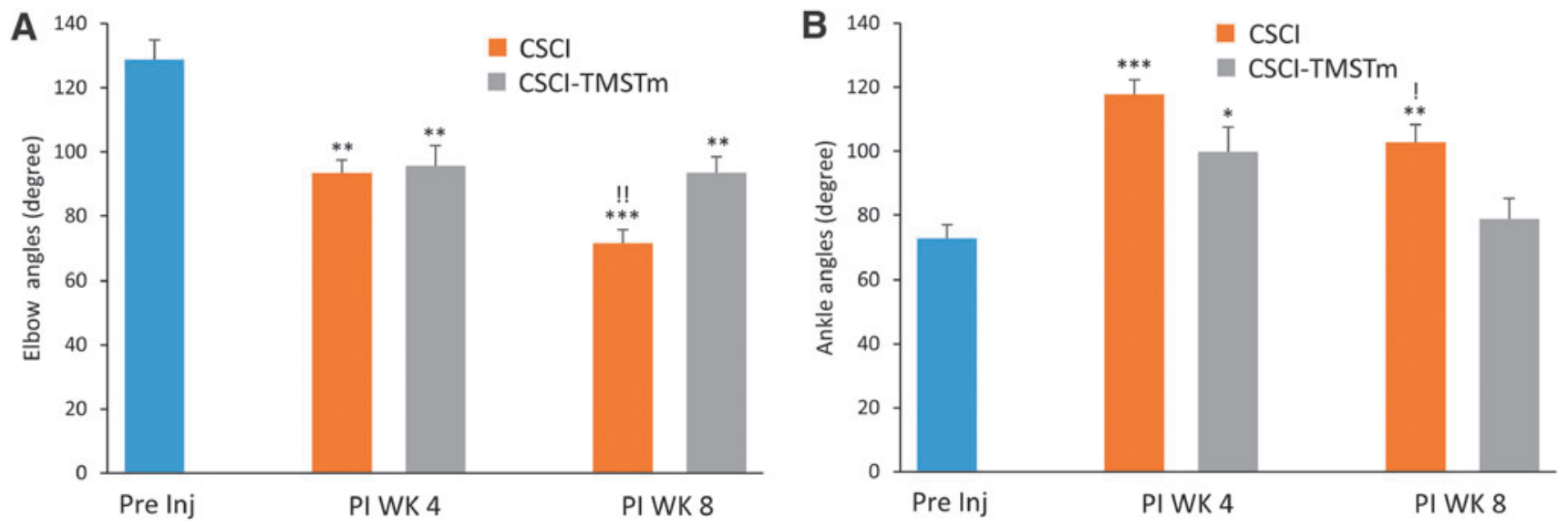

FIG. 5. Group averages of elbow (A) and ankle (B) angles at paw liftoff moment of cervical spinal cord injury (CSCI) and CSCI-transcranial magnetic stimulation/treadmill training (TMSTm) groups at pre-injury, post-injury (PI) week 4, and PI week 8 are shown. Both CSCI and CSCITMSTm groups showed significant decreases in the elbow angles at liftoff moment when compared with pre-injury data. At PI week 8 , however, the CSCI untreated group showed significant decreases in elbow angles when compared with the TMSTm group (A). At PI week 4, both CSCI and TMSTm groups showed significant increases in the ankle angles at paw liftoff moment when compared with pre-injury data. At PI week 8, however, the significant increase of the ankle angles was only observed in the CSCI untreated group. Further, the CSCI untreated group also showed significant increase of ankle angle when compared with the TMSTm group at PI week 8 (B). *, $p<0.05, * *, p<0.01, * * *, p<0.001$, compared with pre-injury; !, $p<0.05, ! !, p<0.01$, compared with CSCI-TMSTm.

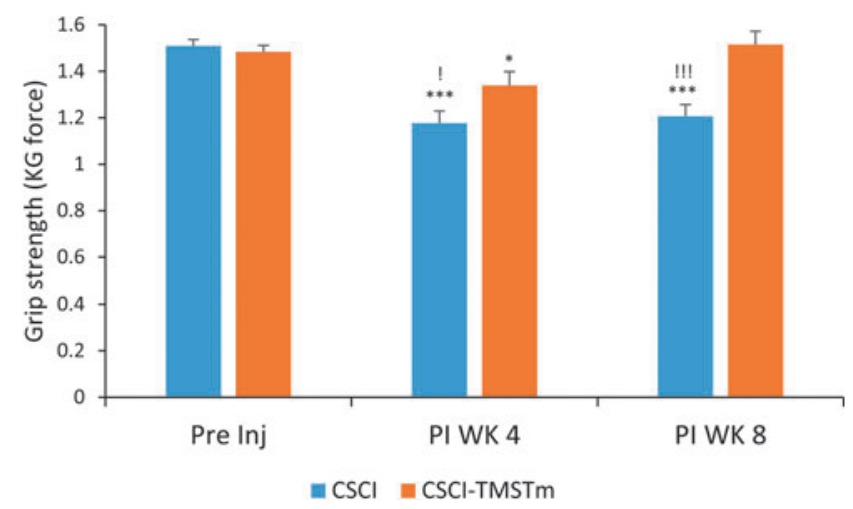

FIG. 6. The transcranial magnetic stimulation/treadmill training (TMSTm) group showed significantly increased grip strength compared with the cervical spinal cord injury (CSCI) untreated group at both post-injury (PI) week 4 and PI week 8 . In addition, the TMSTm group did not show significant difference when compared with pre-injury at PI week $8 . *, p<0.05, * * *, p<0.001$, compared with pre-injury; !, $p<0.05$, !!!, $p<0.001$, compared with CSCI-TMSTm. 
Normal
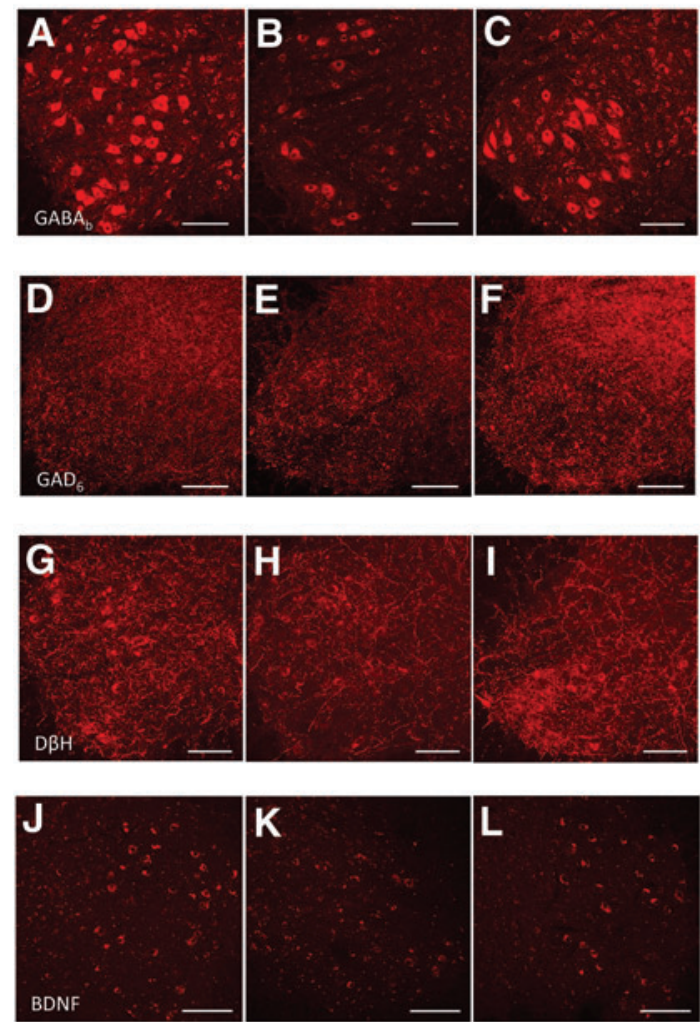

M

$\mathbf{N}$

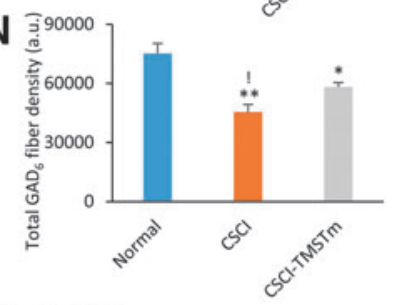

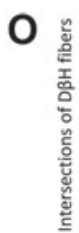

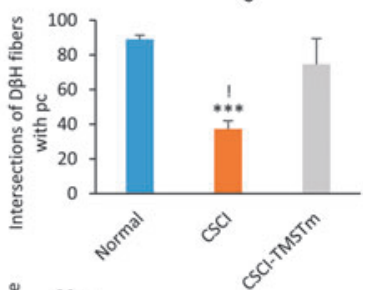

$\mathbf{P}$

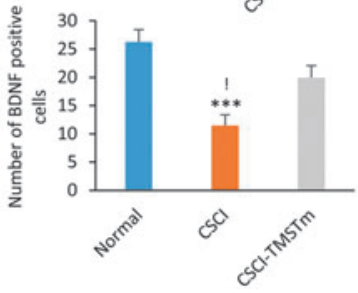

FIG. 7. Immunohistochemical staining of gamma-aminobutyric acid receptor B $\left(\mathrm{GABA}_{\mathrm{b})}(\mathbf{A}-\mathbf{C})\right.$, glutamic acid decarboxylase $\left(\mathrm{GAD}_{6}\right)(\mathbf{D}-\mathbf{F})$, dopamine betahydroxylse $(\mathrm{D} \beta \mathrm{H}) \quad(\mathbf{G}-\mathbf{I})$, and brainderived neurotrophic factor (BDNF) (J-L) in lumbar enlargement of the spinal cord (SC) (L3-4) of normal (A, D, G, J), cervical spinal cord injury (CSCI) (B, E, H, $\mathbf{K})$, and transcranial magnetic stimulation/treadmill training (TMSTm) (C, F, I, L) animals. The gray matter of one side of the SC is shown here. The TMSTm SC specimens exhibited significant upregulation of immunoreactive staining of $\mathrm{GABA}_{\mathrm{b}}(\mathbf{M}), \mathrm{GAD}_{6}(\mathbf{N}), \mathrm{D} \beta \mathrm{H}(\mathbf{O})$, and $\operatorname{BDNF}(\mathbf{P})$ when compared with CSCI untreated samples. Scale bar, $200 \mu \mathrm{m}$. *, $p<0.05, \quad * *, \quad p<0.01, \quad * * *, \quad p<0.001$, compared with normal; !, $p<0.05$, !!, $p<0.01$, compared with CSCI-TMSTm.

A $\mathrm{CSCl}$

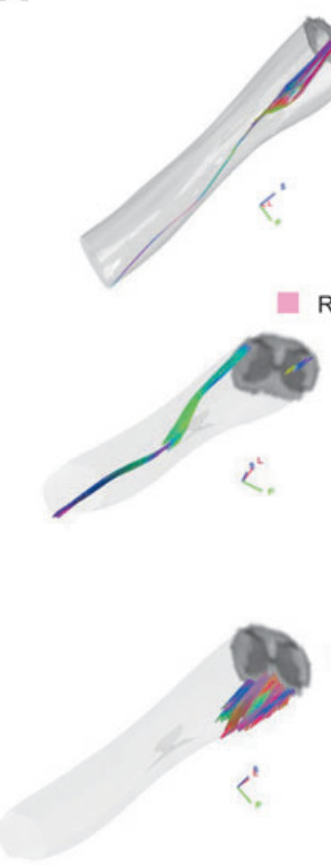

CSCI-TMSTm

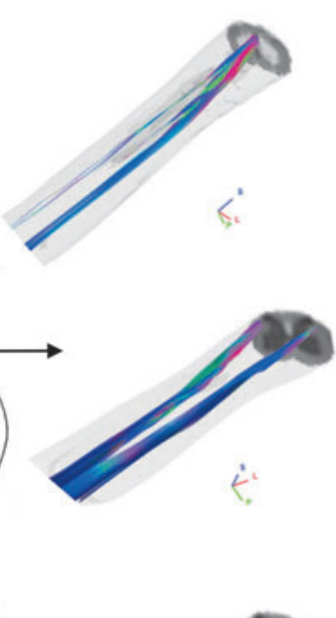

Rostral reticulospinal \& Vestibulospinal tract
Dorsal corticospinal tract
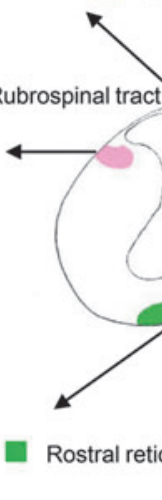

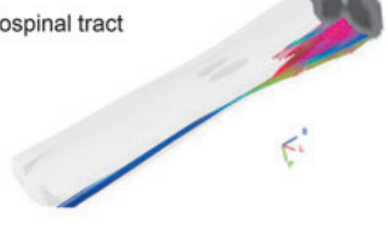

B

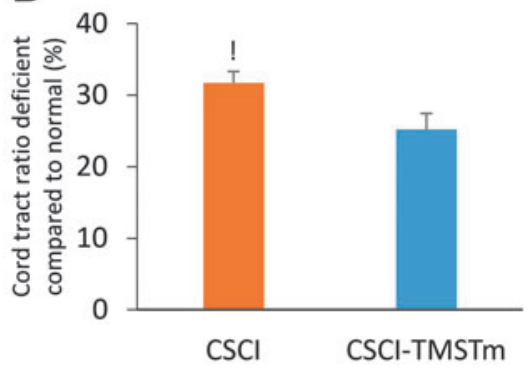

FIG. 9. The diffusion tractography of the dorsal corticospinal tract, rubrospinal tract, and reticulospinal and vestibulospinal tracts are shown in (A). The region of interest selected for each tract is also shown in (A). The transcranial magnetic stimulation/treadmill training (TMSTm) treated spinal cord (SC) showed remarkable sparing and longitudinal continuity of these tracts than those observed in the cervical spinal cord injury (CSCI) controls. Moreover, the TMSTm treated SC showed significant decreases in the deficiency density ratio of spared white matter compared with untreated SC (B). !, $p<0.05$, compared with CSCI-TMSTm. 